\title{
Comprehensive Analysis of Prognostic and Immune Infiltrates for E2F Transcription Factors in Human Pancreatic Adenocarcinoma
}

\author{
Xu-Sheng Liu ${ }^{1 \dagger}$, Yan Gao ${ }^{1 \dagger}$, Chao Liu ${ }^{2 \dagger}$, Xue-Qin Chen ${ }^{3}$, Lu-Meng Zhou ${ }^{1}$, \\ Jian-Wei Yang ${ }^{1}$, Xue-Yan Kui ${ }^{1}$ and Zhi-Jun Pei ${ }^{1,4,5 *}$
}

OPEN ACCESS

Edited by:

Divya P. Kumar,

JSS Academy of Higher Education

and Research, India

Reviewed by:

Ashwini Kumar,

Indian Institute of Technology Delhi,

India

Senthilkumar Rajagopal,

Rayalaseema University, India

*Correspondence:

Zhi-Jun Pei

pzjun1980@yeah.net

${ }^{\dagger}$ These authors have contributed equally to this work

Specialty section:

This article was submitted to Gastrointestinal Cancers,

a section of the journal

Frontiers in Oncology

Received: 16 September 2020 Accepted: 15 December 2020 Published: 02 February 2021

Citation:

Liu X-S, Gao Y, Liu C, Chen X-Q,

Zhou L-M, Yang J-W, Kui $X-Y$ and

Pei Z-J (2021) Comprehensive Analysis of Prognostic and Immune Infiltrates for E2F Transcription Factors

in Human Pancreatic

Adenocarcinoma.

Front. Oncol. 10:606735.

doi: 10.3389/fonc.2020.606735
${ }^{1}$ Department of Nuclear Medicine and Institute of Anesthesiology and Pain, Taihe Hospital, Hubei University of Medicine, Shiyan, China, 2 Medical Imaging Center, Taihe Hospital, Hubei University of Medicine, Shiyan, China, ${ }^{3}$ School of Graduate, Hubei University of Medicine, Shiyan, China, ${ }^{4}$ Hubei Key Laboratory of Embryonic Stem Cell Research, Shiyan, China,

${ }^{5}$ Hubei Key Laboratory of Wudang Local Chinese Medicine Research, Shiyan, China

Background: E2F transcription factors (E2Fs) are a group of genes encoding a family of transcription factors in higher eukaryotes. They are involved in a variety of cellular functions and are up-regulated in many tissues and organs. However, the expression level, genetic variation, molecular mechanism, and biological function of different E2Fs in PAAD and its relationship with the prognosis and immune infiltration in patients with PAAD have not been fully elucidated.

Methods: In this study, we investigated the mRNA expression level, genetic variation, prognostic value and gene-gene interaction network of E2Fs in PAAD using the Oncomine, GEPIA, Kaplan Meier plotter, cBioPortal, GeneMANIA, STRING and Metascape database. Then, the relationship between E2Fs expression and tumor immune invasion was studied by using the TIMER database. Finally, we confirmed the expression of E2Fs in PAAD by IHC.

Results: The transcription levels of E2F1/3/5/8 are obviously up-regulated in PAAD and the high expression of E2F2/3/6/8 was apparently associated with the tumor stage of patients with PAAD. The abnormal expression of E2F1/2/3/4/5/7/8 in PAAD patients is related to the clinical outcome of PAAD patients. We also found that PAAD tissues have higher expression levels of E2F1/3/5/8 compared with adjacent normal tissues. The function of E2Fs and its neighboring genes is mainly related to the transcription initiation of the RNA polymerase II promoter. The functions of E2Fs and its neighboring proteins are mainly related to cell cycle, virus carcinogenesis, FoxO signaling pathway, TGF- $\beta$ signaling pathway, transcriptional disorders in cancer and Wnt signaling pathway. We also found that the expression of E2Fs was significantly correlated with immune infiltrates, including B cells, CD8+ T cells, CD4+T cells, neutrophils, macrophages, and dendritic cells. 


\section{Conclusions: Our study may provide new insights into the choice of immunotherapy targets and prognostic biomarkers in PAAD patients.}

Keywords: pancreatic adenocarcinoma, E2F transcription factors, prognostic value, bioinformatics analysis, tumor microenvironment

\section{INTRODUCTION}

Pancreatic adenocarcinoma (PAAD) is a malignant tumor of the digestive system that is difficult to diagnose and treat (1). Due to the difficulty of early diagnosis, high surgical mortality, poor treatment outcome, the 5-year survival rate of PAAD patients is only $5 \%$, and it has become one of the tumors with high incidence and malignancy in developed countries (2-4). Although PAAD treatment has been partially improved in recent decades, most patients are diagnosed with cancer only in the middle and late stages of cancer because the early symptoms of PAAD patients are not obvious, and there is a lack of biomarkers for early diagnosis. This situation is not effective for surgical resection and drug treatment and ultimately leads to high mortality in patients with PAAD (5). Therefore, it is urgent to find effective biomarkers for early diagnosis and prognosis of PAAD and develop new strategies for targeted therapies for PAAD.

A total of eight E2Fs have been discovered since the first E2F transcription factor (E2F1) was discovered in mammalian cells (6-13). E2Fs are a group of genes encoding a family of transcription factors in higher eukaryotes that are involved in a variety of cellular functions and are expressed in many tissues and organs (14). According to the different functions of E2Fs, they can be divided into two types: transcription activators (E2F1-E2F3) and transcription repressors (E2F4-E2F8). Some studies have found that E2Fs often play a role downstream of mammalian cell cycle signal transduction and affect and control cell proliferation, differentiation, apoptosis, and aging by regulating many target genes (14-17). E2Fs are abnormally expressed in a variety of human malignancies, such as breast cancer (18), ovarian cancer (19), high-grade glioma (20), liver cancer (21), and pancreatic adenocarcinoma (22). Sun F et al. (22) found that overexpression of E2F3 promoted the proliferation of pancreatic tumor cells and the development of pancreatic adenocarcinoma. Lin C et al. (23) suggest that pancreatic tumor growth could be inhibited by inhibiting the expression of E2F5 by miR-1179 overexpression. However, the expression level, genetic variation, molecular mechanism and biological function of different E2Fs in PAAD and its relationship with the prognosis and immune infiltration in patients with PAAD have not been fully elucidated.

Microarray and RNA sequencing technologies have brought huge changes to gene research, and have gradually become a significant part of biological and biomedical research $(24,25)$. In this study, we used various large-scale bioinformatics databases to conduct deeply into and comprehensive bioinformatics exploration of the expression of E2Fs in PAAD. The expression and mutation of different E2Fs in PAAD were analyzed to determine the expression pattern, biological function, prognostic value, and relationship with immune infiltration of E2Fs in PAAD.

\section{MATERIALS AND METHODS}

\section{Ethics Statement}

The study proposal has been approved by the Ethics Committee of Taihe Hospital Affiliated of Hubei University of Medicine (Shiyan, China) (document NO.2020KS042) and conducted in accordance with the principles of the Helsinki Declaration.

\section{Oncomine Analysis}

Oncomine gene expression array dataset (www.oncomine.org) is a publicly accessible online cancer microarray database for analyzing the E2Fs transcription levels in different cancers (26, 27). Student's t test was used to compare the transcription levels of E2Fs in clinical cancer specimens with that in normal controls. The cut-off of $P$-value and fold change were defined as 0.05 and 1.5 , respectively.

\section{GEPIA Dataset Analysis}

Gene Expression Profiling Interactive Analysis (GEPIA, www. gepia.cancer-pku.cn) is a newly developed interactive web server that analyzes RNA sequencing expression data from 9,736 tumors and 8,587 normal samples from the Cancer Genome Atlas (TCGA) and Genotypic Tissue Expression (GTEx) projects using standard processing pipelines (www.gepia.cancer-pku.cn/). GEPIA can provide customizable functions, including differential expression analysis, patient survival analysis, cancer type staging, cancer pathological staging, correlation analysis, similar gene detection and dimensionality reduction analysis (28).

\section{The Kaplan-Meier Plotter Analysis}

The Kaplan-Meier plotter is an online database (www.kmplot. com) that contains gene expression data and survival information for clinical cancer patients $(29,30)$. We used this online tool to assess the prognostic value of E2Fs mRNA expression in PAAD patients and analyzed the overall survival (OS) and recurrence-free survival (RFS) of patients with PAAD. The database divides patient samples into high expression groups and low expression groups according to the median values of E2Fs mRNA expression and validates them by Kaplan-Meier survival curve. Information on number of patients, median values of mRNA expression, 95\% confidence interval (CI), hazard ratio (HR), and $P$-value can be found on the Kaplan-Meier plotter web page. $P$-value $<0.05$ was considered as statistically significant. 


\section{TCGA Data and cBioPortal}

The TCGA database contains sequencing and pathological data for more than 30 different cancers (31). We selected a Pancreatic Adenocarcinoma dataset (TCGA, PanCancer Atlas) that included 184 pathological reports and further analyzed the expression of E2Fs using cBioPortal (www.cbioportal.org) (32, 33). The genomic map includes data for putative copy-number alterations (CNA) from GISTIC, mRNA expression $\mathrm{z}$-scores and mutations. Follow the online instructions of cBioPortal for coexpression and network analysis.

\section{GeneMANIA Analysis}

GeneMANIA (www.genemania.org) is an online analysis tool for deriving hypotheses based on gene functions. GeneMANIA can query and generate a list of genes with similar functions to the target gene and illustrate the relationship between the target gene and the data set by constructing an interactive network (34). In this study, we used GeneMANIA to construct a gene-gene interaction network for E2Fs.

\section{STRINGS Analysis}

STRINGS (www.string-db.org) is an online analysis tool that collects, scores and integrates all publicly available sources of protein-protein interaction (PPI) data and supplements it with calculations and predictions (35). In this study, we performed a PPI network analysis on differentially expressed E2Fs to explore their interactions.

\section{Metascape Analysis}

Metascape (www.genemania.org) is a free and well-maintained online bioinformatics database for Gene Ontology (GO) and Kyoto Encyclopedia of Genes and Genomes (KEGG) enrichment analysis (36). In this study, we used Metascape for functional annotation and pathway enrichment analysis of E2Fs and neighboring genes significantly associated with E2Fs. Only terms with $P$-value $<0.01$, minimum count $>3$, and enrichment factor $>1.5$ were considered as significant.

\section{TIMER Analysis}

TIMER (www.cistrome.shinyapps.io) is a convenient and accurate online analysis tool that can infer the abundance of tumor-infiltrating immune cells from gene expression profiles and evaluate their clinical impact (37). In this study, we used TIMER to assess the correlation between E2Fs expression levels and immune cell infiltration and to assess the correlation between clinical outcomes and immune cell infiltration and E2Fs expression.

\section{Immunohistochemistry}

Clinical samples were obtained from 56 patients with PAAD who were surgically treated at Taihe Hospital Affiliated of Hubei University of Medicine from January 2017 to December 2018. The PAAD tissue and the paired adjacent tissues were prepared into $3 \mu \mathrm{m}$ paraffin sections and incubated with rabbit polyclonal antibodies of E2F1, E2F2, E2F3, E2F4, E2F5, E2F6, E2F7, and E2F8 (1:150, Abcam, USA) at $4^{\circ}$ overnight in a refrigerator. The sections were coupled with secondary antibody labeled with horseradish peroxidase (1:400, Abcam, USA) at room temperature for $1.5 \mathrm{~h}$, then each section was stained with DAB reagent, and finally counterstained with hematoxylin.

\section{RESULTS}

\section{Transcriptional Levels of E2Fs in PAAD Patients}

Eight E2Fs are widely found in mammalian cells but are expressed abnormally in different tumor tissues. We used the Oncomine database to compare the mRNA expression of E2Fs in different cancer and normal tissue samples (Figure 1). ONCOMINE analysis showed that the transcriptional levels of E2F1/3/5/7/8 were up-regulated in PAAD patients. In the Buchholz dataset (38), the transcriptional levels of E2F1 in PAAD were significantly higher than normal tissues, with a fold change of $1.759(P=0.003)$. In three datasets, E2F3 transcription levels were significantly up-regulated in PAAD patients. In the Logsdon dataset (39), the expression of E2F3 in the two groups of PAAD was significantly up-regulated compared with normal samples, with fold changes of $1.551(P=$ $0.001)$ and $1.963(P=4.87 \mathrm{E}-5)$, respectively (Table 1$)$. In the Segara dataset (40), the expression of E2F3 increased in PAAD with a fold change of 1.533 and $(P=4.25 \mathrm{E}-4)$. In the Badea dataset (41), compared with normal samples, the expression of E2F3 increased in PAAD with a fold change of $2.519(P=6.33 \mathrm{E}$ 9). In the Grutzmann dataset (42), the expression of E2F5/7/8 was significantly increased in PAAD, with fold changes of 1.603 $(P=0.009), 4.181(P=7.70 \mathrm{E}-4)$, and $4.334(P=0.002)$, respectively. In the Ishikawa (43) and Pei datasets (44), the expression levels of E2F7 and E2F8 in PAAD were significantly higher than normal tissues, with fold changes of E2F7 of $1.766(P=$ $1.13 \mathrm{E}-4)$ and $2.029(P=2.31 \mathrm{E}-6)$, and fold changes of E2F8 of $2.063(P=0.007)$ and $3.028(P=2.67 \mathrm{E}-8)$, respectively. In addition, ONCOMINE analysis revealed no significant contrast in the transcriptional levels of E2F2, E2F4, and E2F6 between pancreatic adenocarcinoma and normal samples.

\section{Relationship Between the Transcriptional Levels of E2Fs and the Clinicopathological Parameters of PAAD Patients}

We used the GEPIA dataset to compare the transcriptional levels of E2F factors between PAAD and normal simples (Figure 2). Studies have shown that the transcriptional levels of E2F1/3/8 in PAAD tissues were significantly higher than normal pancreatic tissues, while the transcriptional levels of E2F2/4/5/6/7 were not significantly contrast between PAAD tissues and normal pancreatic simples. We also studied the relationship between mRNA expression levels of E2Fs and tumor stage in PAAD patients by using the GEPIA dataset. The results showed that the expression levels of E2F2, E2F3, E2F6, and E2F8 are significantly correlated with the tumor stage of PAAD patients, while the expression levels of E2F1, E2F4, E2F5 and E2F7 are not correlated with the tumor stage of PAAD patients. (Figure 3). 


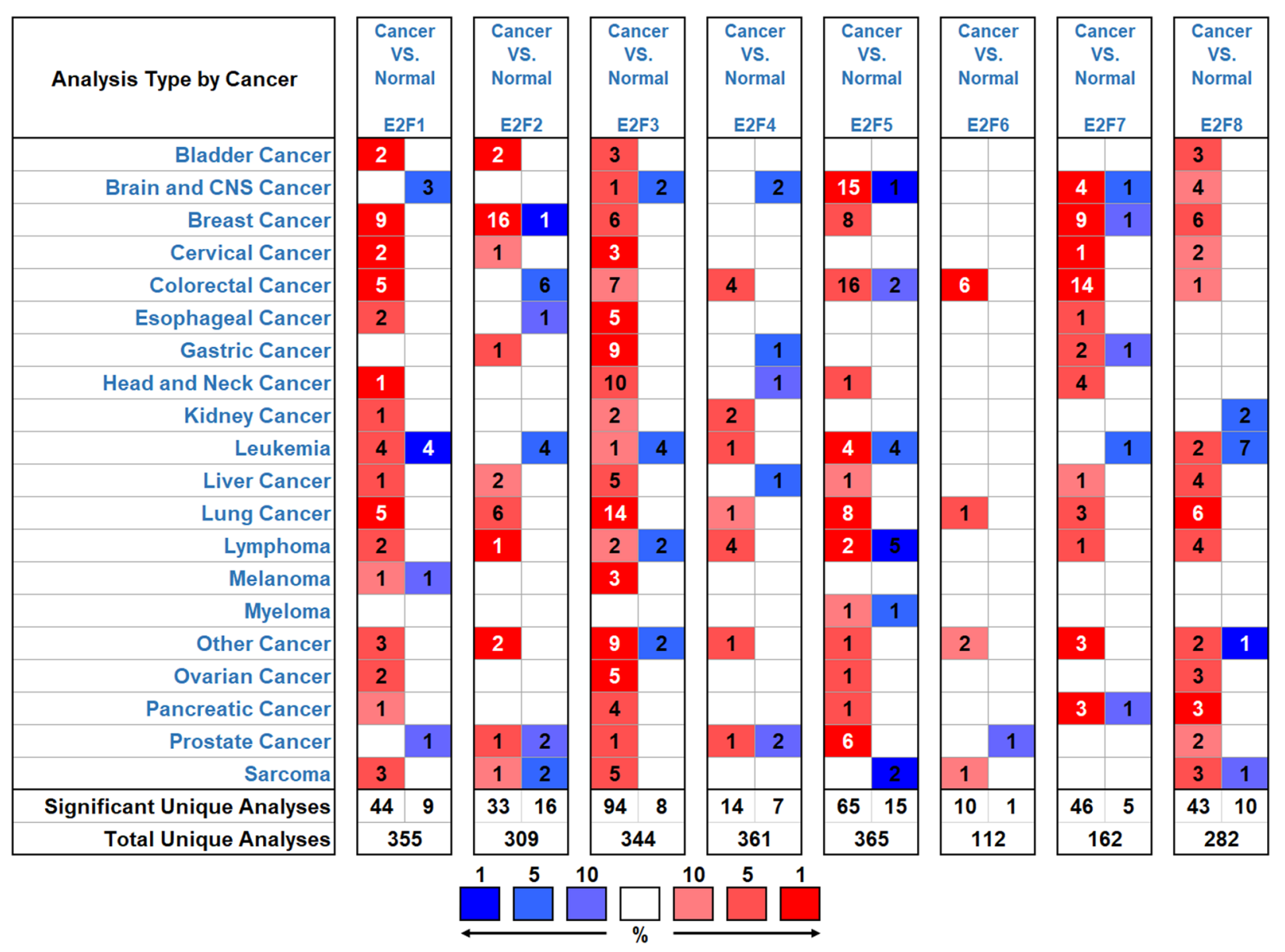

FIGURE 1 | The transcription levels of E2F factors in different types of cancers (Oncomine). The panel shows the numbers of datasets with statistically significant mRNA over-expression (red) or downregulated expression (blue) of E2F factors. The threshold was designed with the following parameters: fold change of 1.5 and $P$-value of 0.05 .

TABLE 1 | The Significant Changes of E2F Expression in Transcription Level between Different Types of Pancreatic adenocarcinoma and Normal Pancreatic Tissues (Oncomine).

\begin{tabular}{|c|c|c|c|c|c|}
\hline & Types of PAAD vs. normal & Fold change & $t$-test & $P$-value & Ref \\
\hline E2F2 & NA & NA & NA & NA & NA \\
\hline \multirow{3}{*}{ E2F3 } & Pancreatic Adenocarcinoma & 1.963 & 5.630 & $4.87 \mathrm{E}-5$ & [39] \\
\hline & Pancreatic Carcinoma & 1.533 & 4.383 & $4.25 \mathrm{E}-4$ & [40] \\
\hline & Pancreatic Ductal Adenocarcinoma & 2.519 & 6.677 & $6.33 \mathrm{E}-9$ & [41] \\
\hline E2F4 & NA & NA & NA & NA & NA \\
\hline \multirow[t]{3}{*}{ E2F7 } & Pancreatic Ductal Adenocarcinoma & 1.766 & 4.018 & $1.13 \mathrm{E}-4$ & [43] \\
\hline & Pancreatic Ductal Adenocarcinoma Epithelia & 4.181 & 3.680 & 7.70E-4 & [42] \\
\hline & Pancreatic Carcinoma & 2.029 & 5.188 & $2.31 \mathrm{E}-6$ & [44] \\
\hline \multirow[t]{3}{*}{ E2F8 } & Pancreatic Ductal Adenocarcinoma Epithelia & 4.334 & 3.413 & 0.002 & [42] \\
\hline & Pancreatic Carcinoma & 3.028 & 6.769 & 2.67E-8 & [44] \\
\hline & Pancreatic Ductal Adenocarcinoma & 2.063 & 2.543 & 0.007 & [43] \\
\hline
\end{tabular}

NA, not available. 

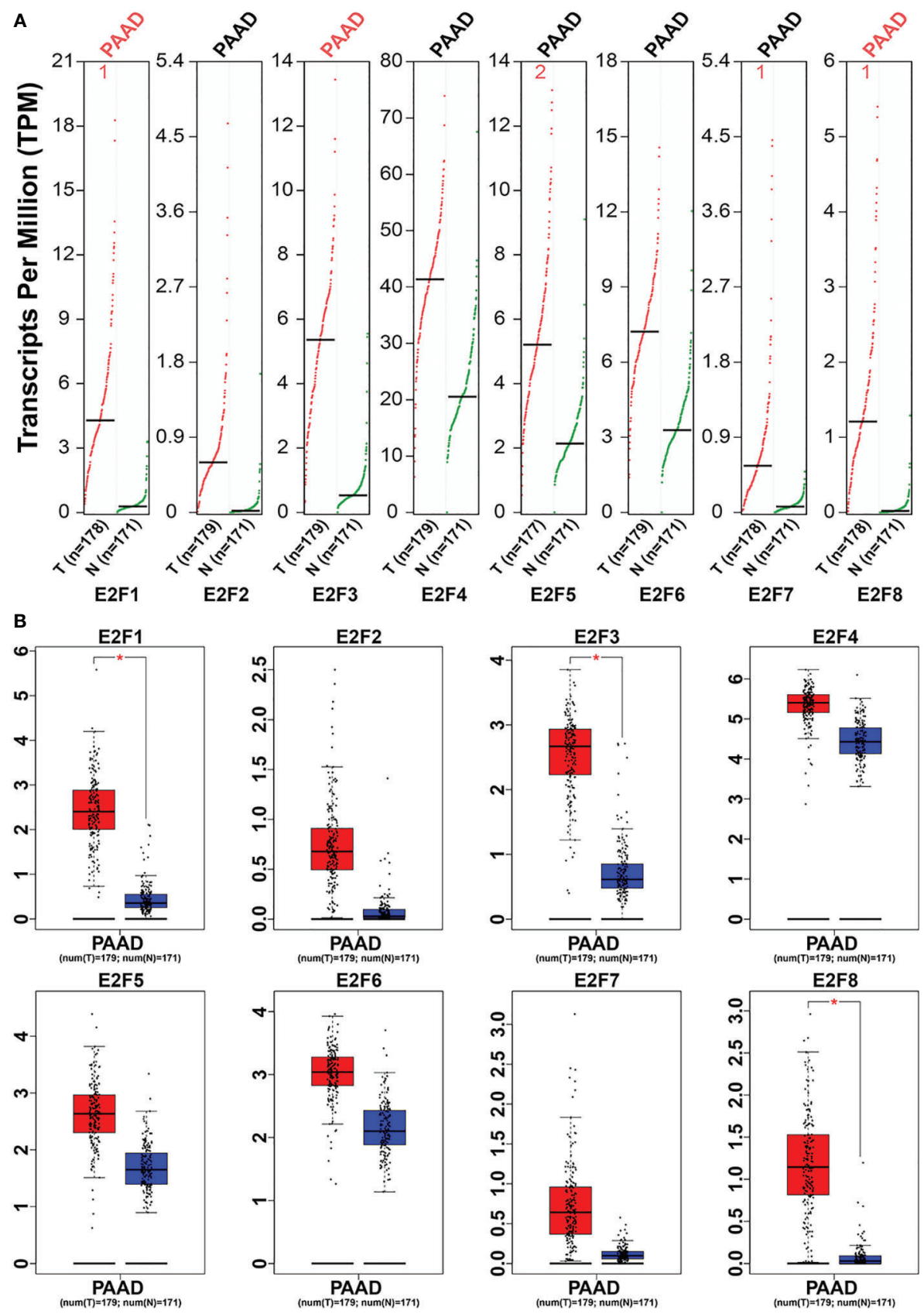

FIGURE 2 | The expression of E2Fs in PAAD (GEPIA),

We used immunohistochemistry (IHC) to detect the protein expression of E2Fs in PAAD and its paired adjacent tissues. We found that the protein levels of E2F1, E2F3, E2F5, and E2F8 are higher in PAAD tissues than in the adjacent tissues (Figure 4).

\section{The Prognostic Value of E2Fs in PAAD Patients}

To evaluate the value of E2Fs at different transcription levels in the progression of PAAD, we evaluated the correlation between E2Fs at different transcription levels and clinical outcome using
Kaplan-Meier plotter analysis. The RFS curve is shown in Figure 5. PAAD patients with low transcription levels of E2F1/2/3/5 were significantly associated with longer RFS. In contrast, PAAD patients with high transcription levels of E2F4 and E2F8 were significantly related with longer RFS, while transcription levels of E2F6 and E2F7 were not related to RFS in PAAD patients. The value of E2Fs at different transcription levels in overall survival of PAAD patients was also evaluated. The studies showed that PAAD with low expression of E2F1/2/3/5 was significantly associated with longer OS. In contrast, PAAD patients with 

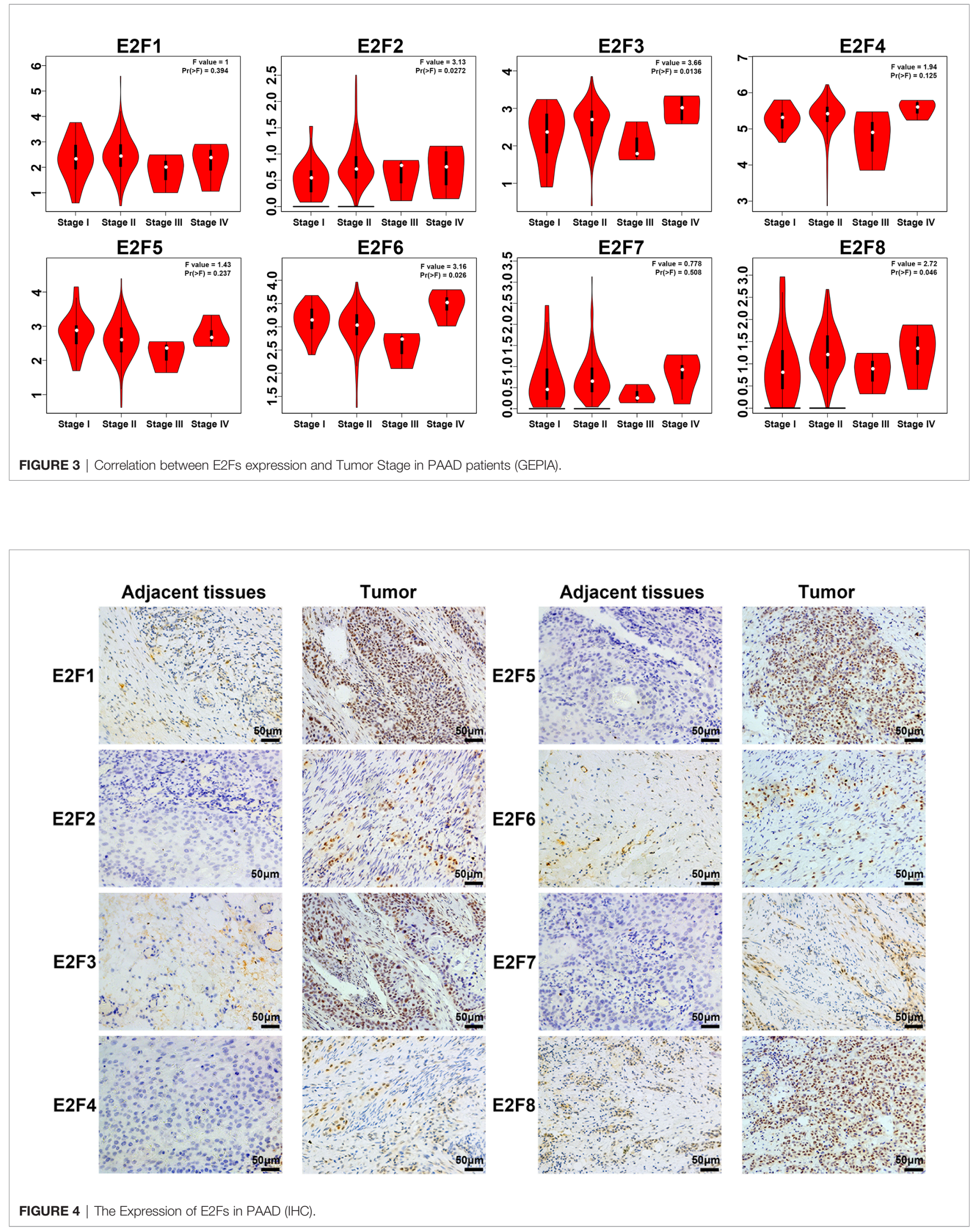


\section{Recurrence-Free Survival (RFS)}
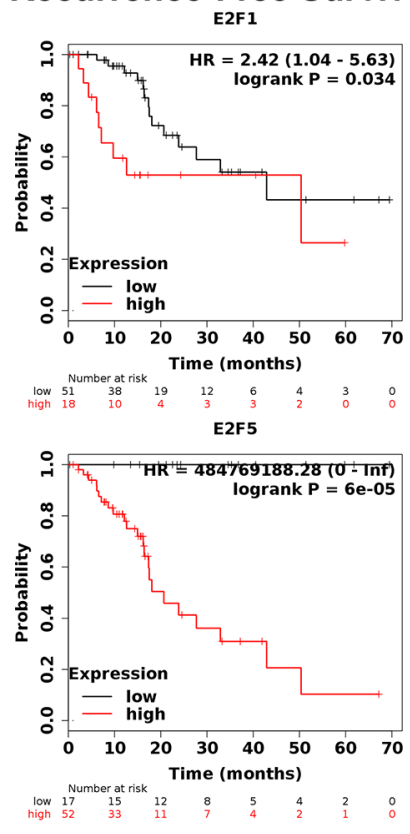

E2F2

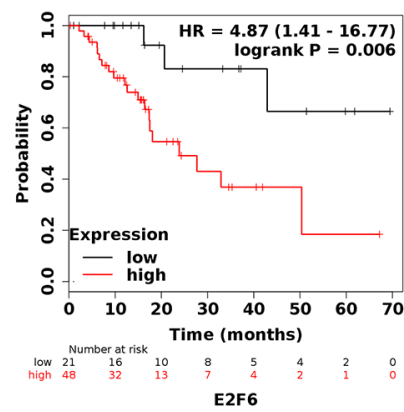

E2F6

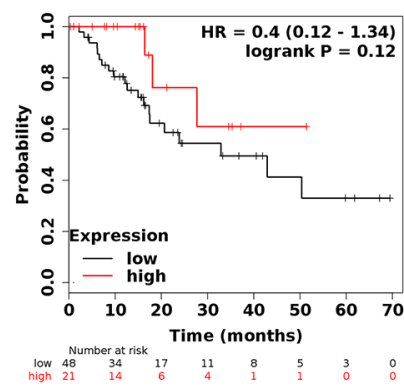

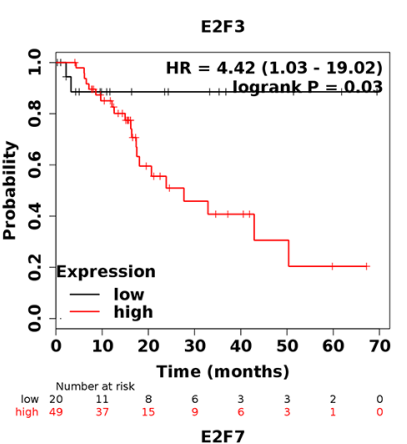

E2F7

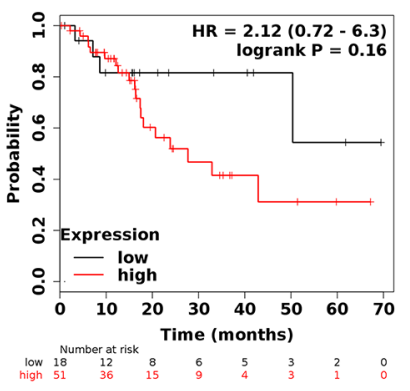

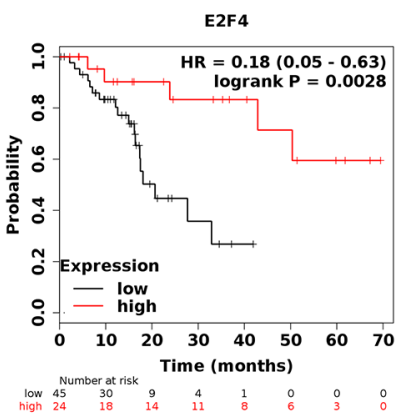

E2F8

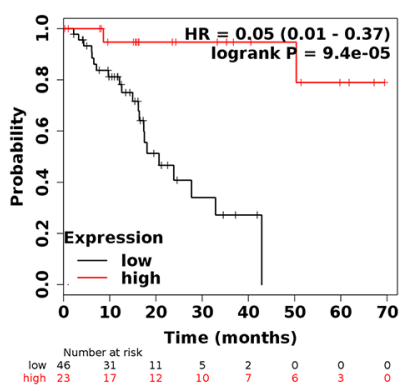

FIGURE 5 | The prognostic value of different expressed E2Fs in PAAD patients in the RFS curve (Kaplan-Meier plotter).

high mRNA expression of E2F4/7/8 were significantly related with longer OS, while E2F6 transcription levels were not associated with OS in PAAD patients (Figure 6).

\section{Genetic Alteration, Co-expression, Neighbor Gene Network, and Interaction Analyses of E2Fs in PAAD Patients}

We used the cBioPortal online tool to analyze changes and correlations of E2Fs in PAAD. Among 168 PAAD patients, E2Fs were changed in 65 samples (38.69\%), and two or more changes were detected in eight samples (4.76\%) (Figure 7A). According to the TCGA data set, the highest genetic variation rate in E2Fs is E2F5 (10\%), the lowest mutation rate is E2F7 (4\%), and the others are E2F1 (10\%), E2F2 (6\%), E2F3 (8\%), E2F4 (8\%), E2F6 (7\%), and E2F8 (8\%) (Figure 7B). Evaluation of the mutual exclusion between the eight E2Fs genes in the TCGA PAAD cohort showed that there are co-expressed relationships between E2F1 and E2F2, E2F1 and E2F4, E2F2 and E2F4, E2F2 and E2F8, E2F3 and E2F5, E2F4 and E2F8 $(P<0.05)$, E2F2 with E2F3, six, seven and E2F6 with E2F7, eight are highly mutually exclusive but not statistically significant $(P>0.05)$, probably due to the small sample size (Figure 7C).

A GGI network of eight E2Fs was constructed, and their functions were analyzed using the GeneMANIA database (Figure 7D). The eight central nodes of E2Fs are surrounded by 20 nodes representing genes that are strongly associated with E2Fs in shared protein domains, physical interactions, colocalization, co-expression, prediction, genetic interactions, and pathway. The top five genes most associated with E2Fs are TFDP2 (transcription factor Dp-2), TFDP1 (transcription factor Dp-1), TFDP3 (transcription factor Dp-3), COPS4 (COP9 signalosome subunit 4), and PSMD12 (proteasome 26S subunit, non-ATPase 12). Among them, TFDP2 is related to E2F1,2,3,4,6 in terms of physical interaction and has a pathway relationship with E2F1 and E2F4. TFDP1 is related to $\mathrm{E} 2 \mathrm{~F} 1,2,3,4,5,6$ in terms of physical interaction, and it is related to genetic interaction in E2F2. However, TFDP2, TFDP1, TFDP3, COPS4, PSMD12, and E2F1/2/3/4/5/6/7/8 all have shared protein domains. Further functional analysis showed that these genes indicated the greatest correlation with transcription initiation from RNA polymerase II promoter $($ FDR $=7.28 \mathrm{E}-8)$. In addition, these genes were correlated with DNA-templated transcription, initiation, core promoter binding, G1/S transition of mitotic cell cycle, regulatory region DNA binding, DNA integrity checkpoint and regulatory region nucleic acid binding activity.

\section{PPI and Functional Enrichment Analysis of E2Fs in PAAD Patients}

We performed a PPI network analysis of E2Fs at different transcription levels using STRING to study the potential interactions between them. As shown in Figure 8A, the PPI network diagram contains eight E2Fs proteins and 20 proteins that are closely related to E2Fs.

In this study, we used Metascape to perform functional annotation and pathway enrichment analysis of E2Fs and their adjacent genes. The first 20 items of GO enrichment are mainly distributed in the biological process (11 items), the molecular function (six items) and the cell component (three items) (Figures 8B, C and Table 2). Three of the first five projects 


\section{Overall Survival (OS)}
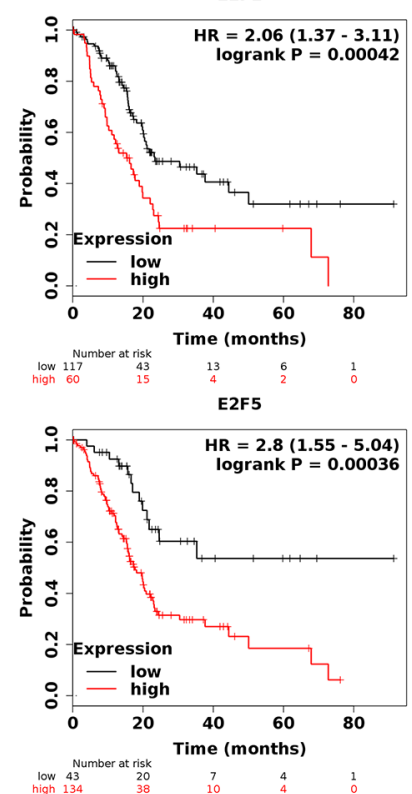
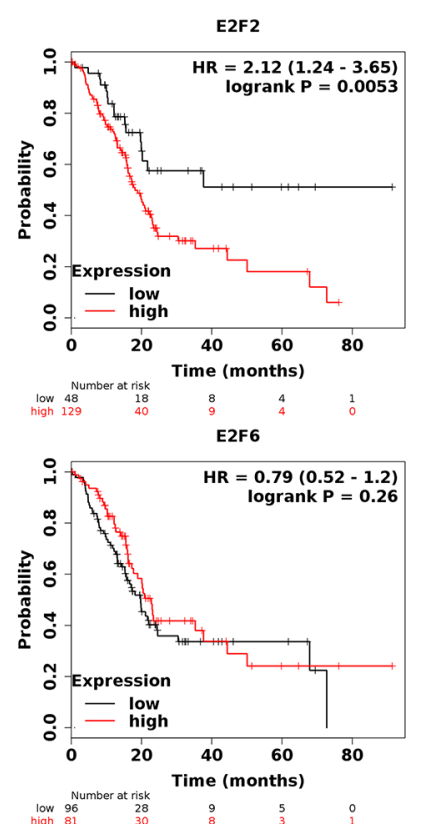
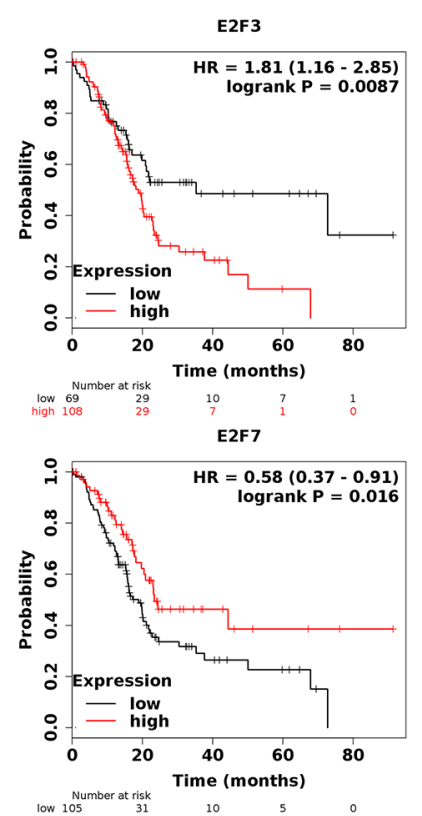

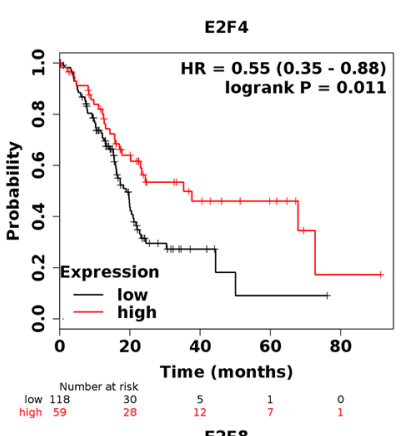

E2F8

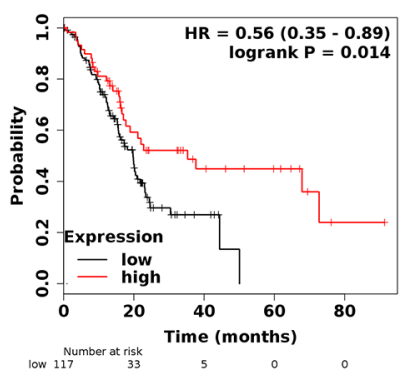

FIGURE 6 | The prognostic value of different expressed E2Fs in PAAD patients in the OS curve (Kaplan-Meier plotter).

are in the biological process, which are cell cycle G1/S phase transition, regulation of transcription involved in G1/S transition of mitotic cell cycle, and negative regulation of G0 to G1 transition, and the other two are cyclin-dependent protein kinase holoenzyme complex and transcription factor binding.

The first 13 KEGG pathways of E2Fs and its adjacent genes are illustrated in Figures 8D, E and Table 3. Among them, cell cycle, transcriptional misregulation in cancer, FoxO signaling pathway, TGF-beta signaling pathway, viral carcinogenesis, and Wnt signaling pathway are significantly associated with the occurrence and development of various tumors and are also involved in the tumorigenesis of PAAD.

\section{The Relationship Between E2Fs Expression Levels and Immune Infiltration Levels in PAAD}

TIMER online analysis tool is used to evaluate the relationship between the transcription level of E2Fs and the level of immune infiltration in PAAD. It was found that E2Fs are involved in inflammatory response and immune cell infiltration, which affect the clinical outcome of PAAD patients. The analysis results are shown in Figure 9. E2F2 expression was positively correlated with infiltration of B cells, neutrophil, and dendritic cells (Figure 9B). E2F3, E2F4, E2F5, and E2F6 expressions were positively correlated with the infiltration of $\mathrm{B}$ cells, CD8+ $\mathrm{T}$ cells, macrophage, neutrophil, and dendritic cells, and E2F6 expressions were also positively correlated with the infiltration of CD4+ T cells (Figures 9C-F). E2F7 expression was positively correlated with infiltration of CD8 $+\mathrm{T}$ cells and dendritic cells, while it was negatively correlated with infiltration of $\mathrm{CD} 4+\mathrm{T}$ cells (Figure 9G). E2F8 expression was negatively correlated with infiltration of CD4+ T cells, while it was positively correlated with infiltration of B cells and dendritic cells (Figure 9H). These studies indicate that the level of E2Fs expression is associated to the level of immune infiltration in PAAD.

We also use the TIMER online tool to proofread B cells, CD8+ $\mathrm{T}$ cells, CD4+ T cells, macrophage, neutrophil, E2F1/2/3/4/5/6/7/ 8 covariate factors and automatically output Cox regression results. These studies indicate that the infiltration of $\mathrm{CD} 8+\mathrm{T}$ cells $(P=0.030)$, neutrophil $(P=0.026)$, and E2F1 $(P=0.015)$ was significantly correlated with the clinical outcomes of patients with PAAD (Table 4).

\section{DISCUSSION}

Studies have shown that the dysregulation of E2Fs is significantly associated to the occurrence and development of many tumors (18-23). E2Fs not only participate in tumor cell proliferation and differentiation, but also affect cell apoptosis and senescence (1417). Although the role of E2Fs in the occurrence and survival prognosis of certain cancers has been elucidated, there have been no reports of different E2Fs expression and role in PAAD. This study is the first study to explore the transcription level, genetic variation, molecular mechanism, and biological function of different E2Fs in PAAD and its relationship with the prognosis and immune infiltration in patients with PAAD through bioinformatics analysis.

Among all members of E2Fs, E2F1 is the earliest found and most researched member. Studies have shown that E2F1 can play 

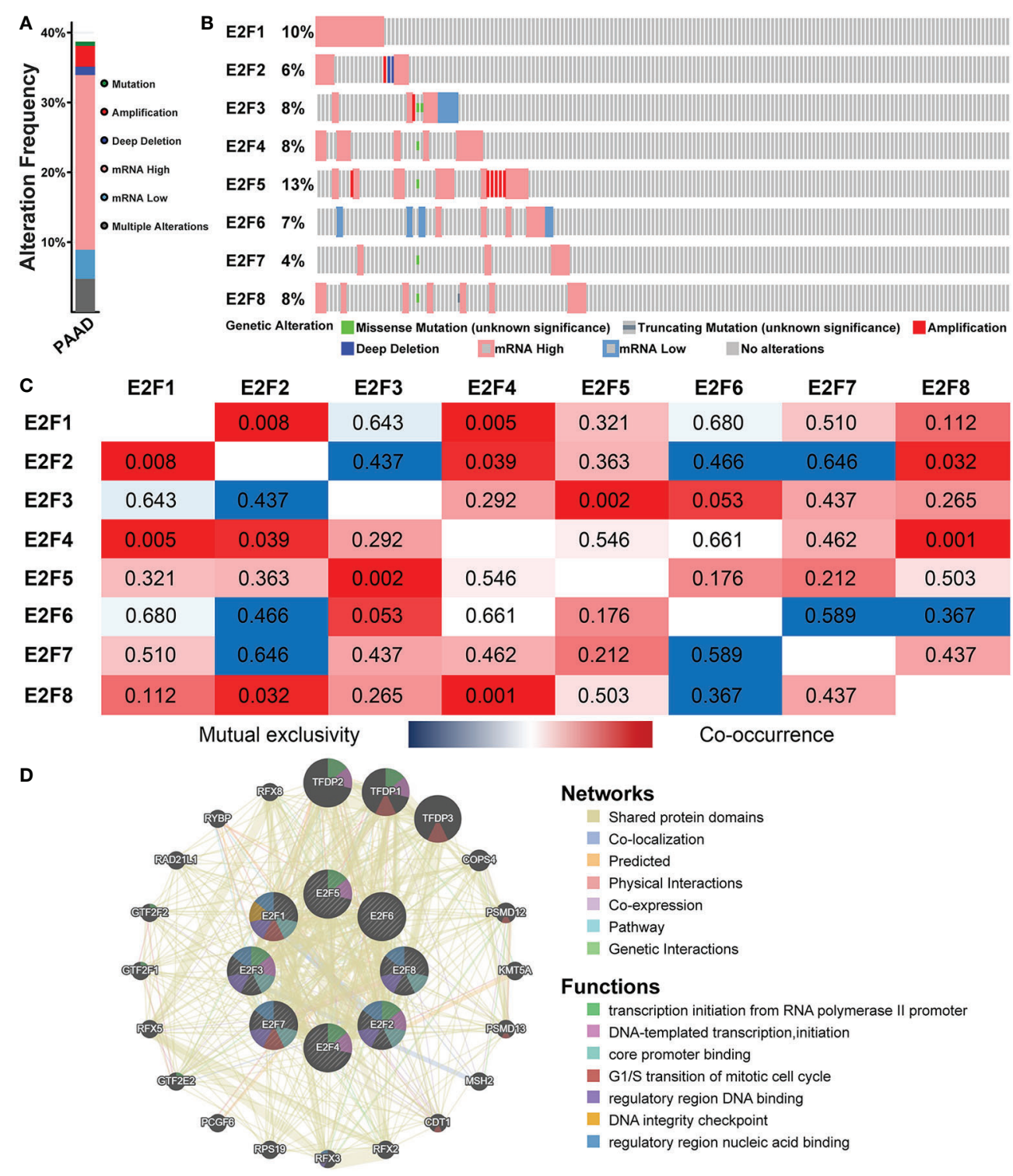

\author{
Networks \\ Shared protein domains
Co-localization \\ Predicted \\ Physical Interactions \\ - Co-expression \\ 11 Pathway \\ - Genetic Interactions
}

\title{
Functions
}

transcription initiation from RNA polymerase II promoter

DNA-templated transcription, initiation

core promoter binding

- G1/S transition of mitotic cell cycle

regulatory region DNA binding

DNA integrity checkpoint

regulatory region nucleic acid binding

FIGURE 7 | Genetic alteration, co-expression, neighbor gene network, and interaction analyses of E2Fs in patients with PAAD (cBioPortal and GeneMANIA). (A) Summary of alterations in different expressed E2Fs in PAAD. (B) OncoPrint visual summary of alteration on a query of E2F family members. (C) Correlation heat map of different expressed E2Fs in PAAD. Red and blue cells indicate co-occurrence and mutual exclusivity, respectively. The numbers in the color blocks represent the P-values. (D) Gene-gene interaction network of different expressed E2Fs. Each node represents a gene, and the size of the node represents the strength of the interaction. The color of the connection lines between nodes represents the type of gene-gene interaction. Node colors indicate the possible functions of the respective genes.

different roles in tumor suppressor genes or oncogenes in different cancers $(45,46)$. However, Ma L et al. (47) found that E2F1 is overexpressed in pancreatic adenocarcinoma tissues, and the up-regulation of E2F1 promotes the proliferation of cancer cells and exerts carcinogenesis. In this study, database analysis found that the transcription levels of E2F1 in human PAAD were higher than in normal tissues, and immunohistochemical staining also verified this result. But the expression of E2F1 in PAAD patients has nothing to do with the tumor stage. Kaplan-
Meier plotter analysis revealed that high E2F1 transcription levels were associated with poor OS and RFS in PAAD patients.

E2F2 plays a significant role in the occurrence and development of PAAD. E2F2, like E2F1, can play a dual role in suppressing and causing cancer $(48,49)$. Yao Z et al. (50) found that miR-214-5p can regulate the expression of E2F2 in PAAD cells to achieve the role of oncogenes. However, in this study, database analysis found no difference in E2F2 expression in PAAD compared to normal tissues. However, the expression of 


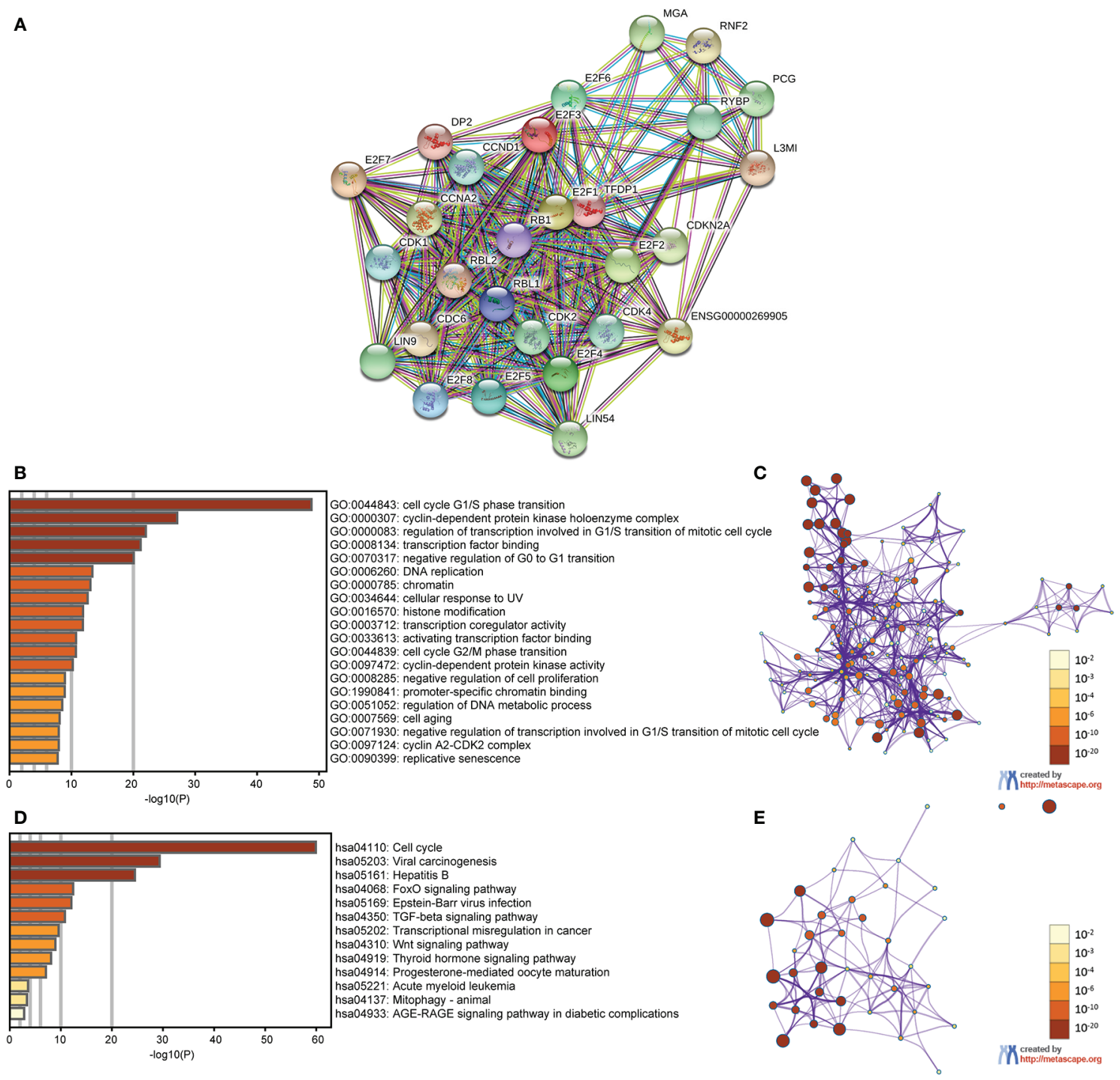

FIGURE 8 | PPI and functional enrichment analysis of E2Fs in patients with PAAD (STRING and Metascape). (A) Protein-protein interaction network of different expressed E2Fs. (B) E2Fs gene ontology (GO) enriched terms, colored by $P$-values. (C) Network of GO enriched terms colored by $P$-value, where terms containing more genes tend to have a more significant $P$-value. (D) E2Fs Kyoto Encyclopedia of Genes and Genomes (KEGG) enriched terms, colored by $P$-values. (E) Network of KEGG enriched terms colored by $P$-value, where terms containing more genes tend to have a more significant $P$-value.

E2F2 in patients with PAAD is related to the stage of the tumor. It was also found that the high transcription levels of E2F2 was related with poor OS and RFS in PAAD patients.

E2F3 is crucial for controlling tumor cell proliferation rate and regulating cell cycle (8). Sun FB et al. (22) found that upregulating E2F3 expression by knocking down MiR-210 promotes tumor cell proliferation and thus the development of pancreatic adenocarcinoma. The analysis of the data and IHC in this study found that the expression levels of E2F3 in human PAAD were higher than in normal tissues, and it was related to tumor stage in PAAD patients. The results of survival analysis indicated that high transcription levels of E2F3 result in worse OS and RFS in patients with PAAD.
E2F4 is an important regulator of cell transformation and proliferation. Studies have shown that over-expressed E2F4 is related to colon cancer, kidney cancer, and lung cancer. However, the analysis of the data in this study found that the transcription levels of E2F4 in PAAD were not different from that in normal tissues and had nothing to do with the tumor stage of PAAD patients. The results of survival analysis indicated that high expression of E2F4 was associated with better OS and RFS in PAAD patients. This result may herald the role of E2F4 as a tumor suppressor gene in PAAD.

E2F5 is an important member of E2Fs. E2Fs have been studied more in other tumors but less in PAAD. In this study, it was found that the expression level of E2F5 in human PAAD is 
TABLE 2 | The GO function enrichment analysis of E2Fs and neighbor genes in PAAD (Metascape).

\begin{tabular}{|c|c|c|c|c|c|c|}
\hline GO & Category & Description & Count & $\%$ & $\log 10(P)$ & $\log 10(q)$ \\
\hline GO:0044843 & GO Biological Processes & cell cycle G1/S phase transition & 33 & 60 & -48.77 & -44.42 \\
\hline GO:0000307 & GO Cellular Components & cyclin-dependent protein kinase holoenzyme complex & 14 & 25.45 & -27.08 & -24.07 \\
\hline GO:0000083 & GO Biological Processes & regulation of transcription involved in $\mathrm{G} 1 / \mathrm{S}$ transition of mitotic cell cycle & 11 & 20 & -22.01 & -19.28 \\
\hline GO:0008134 & GO Molecular Functions & transcription factor binding & 23 & 41.82 & -21.19 & -18.5 \\
\hline GO:0070317 & GO Biological Processes & negative regulation of $\mathrm{G} 0$ to $\mathrm{G} 1$ transition & 11 & 20 & -20.06 & -17.42 \\
\hline GO:0006260 & GO Biological Processes & DNA replication & 13 & 23.64 & -13.39 & -10.89 \\
\hline GO:0000785 & GO Cellular Components & chromatin & 16 & 29.09 & -13.08 & -10.58 \\
\hline GO:0034644 & GO Biological Processes & cellular response to UV & 9 & 16.36 & -12.62 & -10.13 \\
\hline GO:0016570 & GO Biological Processes & histone modification & 14 & 25.45 & -11.88 & -9.42 \\
\hline GO:0003712 & GO Molecular Functions & transcription coregulator activity & 15 & 27.27 & -11.87 & -9.41 \\
\hline GO:0033613 & GO Molecular Functions & activating transcription factor binding & 8 & 14.55 & -10.75 & -8.34 \\
\hline GO:0044839 & GO Biological Processes & cell cycle G2/M phase transition & 11 & 20 & -10.74 & -8.34 \\
\hline GO:0097472 & GO Molecular Functions & cyclin-dependent protein kinase activity & 6 & 10.91 & -10.22 & -7.84 \\
\hline GO:0008285 & GO Biological Processes & negative regulation of cell proliferation & 14 & 25.45 & -8.95 & -6.61 \\
\hline GO:1990841 & GO Molecular Functions & promoter-specific chromatin binding & 6 & 10.91 & -8.92 & -6.59 \\
\hline GO:0051052 & GO Biological Processes & regulation of DNA metabolic process & 11 & 20 & -8.52 & -6.21 \\
\hline GO:0007569 & GO Biological Processes & cell aging & 7 & 12.73 & -8.08 & -5.83 \\
\hline GO:0097124 & GO Cellular Components & cyclin A2-CDK2 complex & 3 & 5.45 & -7.95 & -5.71 \\
\hline GO:0071930 & GO Biological Processes & negative regulation of transcription involved in G1/S transition of mitotic cell cycle & 3 & 5.45 & -7.95 & -5.71 \\
\hline GO:0090399 & GO Biological Processes & replicative senescence & 4 & 7.27 & -7.77 & -5.54 \\
\hline
\end{tabular}

TABLE 3 | The KEEG function enrichment analysis of E2Fs and neighbor genes in PAAD (Metascape).

\begin{tabular}{|c|c|c|c|c|c|c|}
\hline GO & Category & Description & Count & $\%$ & $\log 10(P)$ & $\log 10(q)$ \\
\hline hsa04110 & KEGG Pathway & Cell cycle & 32 & 58.18 & -59.93 & -57.24 \\
\hline hsa05203 & KEGG Pathway & Viral carcinogenesis & 21 & 38.18 & -29.32 & -26.93 \\
\hline hsa05161 & KEGG Pathway & Hepatitis B & 17 & 30.91 & -24.49 & -22.4 \\
\hline hsa04068 & KEGG Pathway & FoxO signaling pathway & 10 & 18.18 & -12.4 & -10.96 \\
\hline hsa05169 & KEGG Pathway & Epstein-Barr virus infection & 11 & 20 & -12.06 & -10.67 \\
\hline hsa04350 & KEGG Pathway & TGF-beta signaling pathway & 8 & 14.55 & -10.79 & -9.42 \\
\hline hsa05202 & KEGG Pathway & Transcriptional misregulation in cancer & 9 & 16.36 & -9.56 & -8.23 \\
\hline hsa04310 & KEGG Pathway & Wnt signaling pathway & 8 & 14.55 & -8.92 & -7.61 \\
\hline hsa04919 & KEGG Pathway & Thyroid hormone signaling pathway & 7 & 12.73 & -8.08 & -6.79 \\
\hline hsa04914 & KEGG Pathway & Progesterone-mediated oocyte maturation & 6 & 10.91 & -7.08 & -5.8 \\
\hline hsa05221 & KEGG Pathway & Acute myeloid leukemia & 3 & 5.45 & -3.57 & -2.43 \\
\hline hsa04137 & KEGG Pathway & Mitophagy - animal & 3 & 5.45 & -3.36 & -2.24 \\
\hline hsa04933 & KEGG Pathway & AGE-RAGE signaling pathway in diabetic complications & 3 & 5.45 & -2.82 & -1.74 \\
\hline
\end{tabular}

significantly different from that of normal tissues, but the expression level has nothing to do with the tumor stage of PAAD patients. However, the results of survival analysis indicated that high transcription levels of E2F5 lead to worse OS and RFS in PAAD patients.

No article on the role of E2F6 in PAAD has been published. The results from our analysis found that although E2F6 is related to tumor stage in PAAD patients, the expression of E2F6 is not different between PAAD and normal tissues and has no effect on the survival prognosis of PAAD.

Some studies indicate that E2F7 is up-regulated in many tumors, but there is no relevant report on the role of E2F7 in PAAD. The analysis of this study found that the transcription levels of E2F7 were not different between PAAD and normal tissues, and had no effect on tumor stage. However, survival analysis showed that high expression of E2F7 leads to better OS in patients with PAAD.

As for E2F8, although its role in PAAD has not been reported, our study found that the transcription level of E2F8 in PAAD is significantly higher than in normal tissues, and it is closely related to the tumor stage of PAAD patients. However, survival analysis showed that high transcription level of E2F8 leads to worse OS and RFS in PAAD patients.

Furthermore, a high mutation rate $(38.69 \%)$ of E2Fs was noticed in PAAD patients, and a mutually exclusive or cooccurring connection was found between differentially expressed E2Fs, indicating that these cytokines play an antagonistic or synergistic role in the occurrence and development of PAAD. We also constructed a GGI network of E2Fs and its neighboring genes, and found that these genes showed the greatest correlation with the transcription initiation of the RNA polymerase II promoter. Then we analyzed the function of E2Fs and its neighboring proteins by GO enrichment analysis and KEGG pathway enrichment. Studies have found that the functions of these proteins are mainly related to the cell cycle, viral carcinogenesis, FoxO signaling pathway, TGF-beta signaling pathway, transcriptional misregulation in cancer and Wnt signaling pathway, and these pathways are significantly 

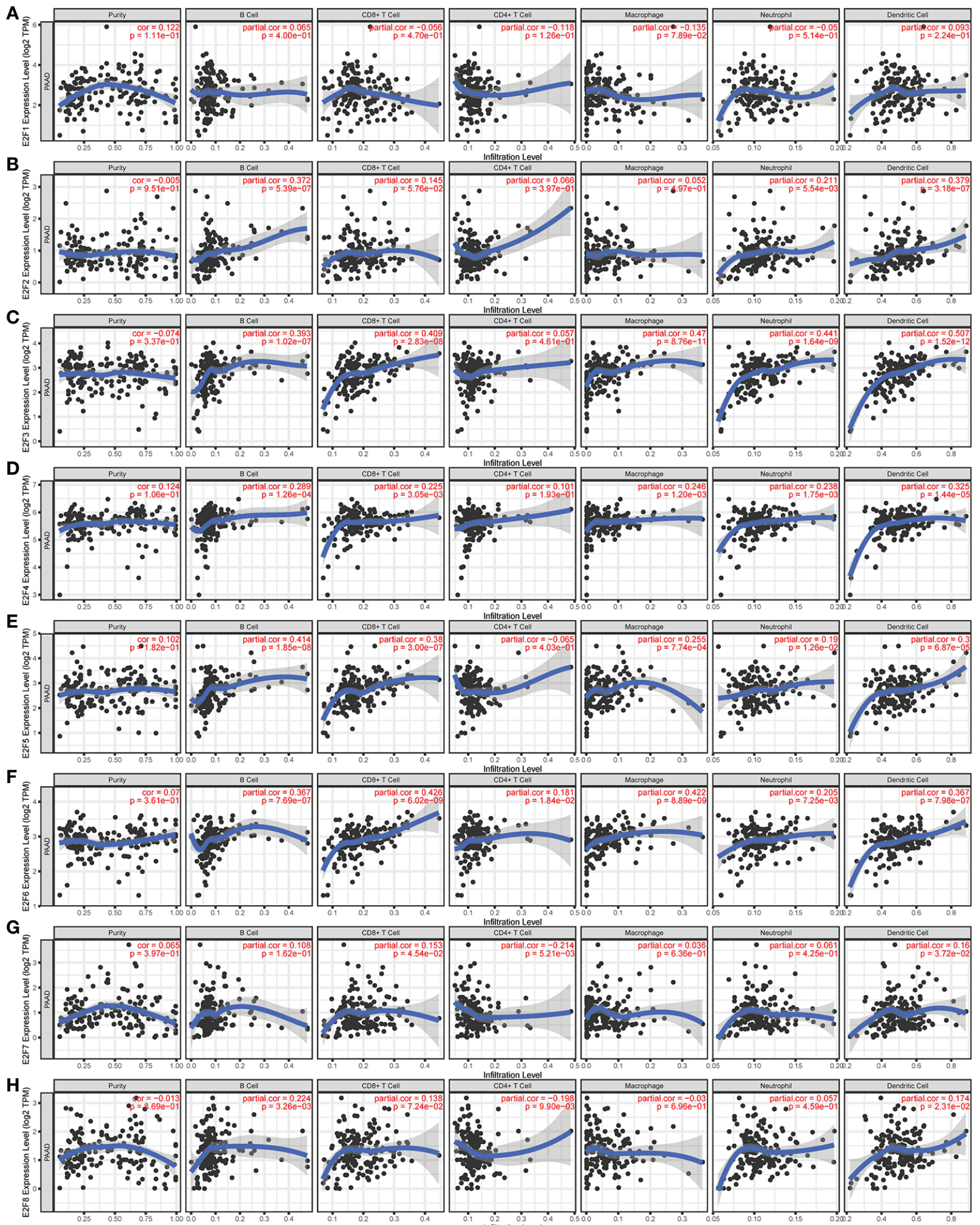

FIGURE 9 | The Relationship between E2Fs Expression Levels and Immune Infiltration Levels in PAAD (TIMER). The correlation between the abundance of immune cell and the expression of (A) E2F1, (B) E2F2, (C) E2F3, (D) E2F4, (E) E2F5, (F) E2F6, (G) E2F7, (H) E2F8 in PAAD. 
TABLE 4 | The cox proportional hazard model of CXC chemokines and six tumor-infiltrating immune cells in PAAD (TIMER).

\begin{tabular}{|c|c|c|c|c|c|c|}
\hline & coef & HR & 95\%Cl_I & 95\%Cl_u & $P$-value & sig \\
\hline B_cell & 3.833 & 46.203 & 0.193 & $1.11 \mathrm{E}+04$ & 0.170 & \\
\hline CD8_Tcell & 7.455 & $1,728.250$ & 2.090 & $1.43 \mathrm{E}+06$ & 0.030 & * \\
\hline CD4_Tcell & -2.800 & 0.061 & 0.000 & $6.44 \mathrm{E}+01$ & 0.431 & \\
\hline Macrophage & -5.591 & 0.004 & 0.000 & $1.32 \mathrm{E}+00$ & 0.062 & \\
\hline Neutrophil & 15.292 & $437,7762.585$ & 6.027 & $3.18 \mathrm{E}+12$ & 0.026 & * \\
\hline Dendritic & -1.940 & 0.144 & 0.005 & $4.29 \mathrm{E}+00$ & 0.263 & \\
\hline E2F1 & 0.576 & 1.780 & 1.120 & $2.83 \mathrm{E}+00$ & 0.015 & * \\
\hline E2F2 & -0.481 & 0.618 & 0.301 & $1.27 \mathrm{E}+00$ & 0.189 & \\
\hline E2F3 & 0.188 & 1.207 & 0.704 & $2.07 \mathrm{E}+00$ & 0.494 & \\
\hline E2F4 & -0.578 & 0.561 & 0.252 & $1.25 \mathrm{E}+00$ & 0.157 & \\
\hline E2F5 & -0.126 & 0.882 & 0.548 & $1.42 \mathrm{E}+00$ & 0.604 & \\
\hline E2F6 & -0.695 & 0.499 & 0.223 & $1.12 \mathrm{E}+00$ & 0.091 & \\
\hline E2F7 & 0.143 & 1.154 & 0.733 & $1.82 \mathrm{E}+00$ & 0.535 & \\
\hline
\end{tabular}

${ }^{*} P<0.05$.

associated with the occurrence and development of PAAD. These data indicate that differentially expressed E2Fs in PAAD are potential targets for drug therapy.

Another important result of this study is that the transcription levels of E2Fs is closely correlated with various levels of immune infiltration in PAAD. The transcription level of E2F2 was positively correlated with infiltration of B cells, neutrophil, and dendritic cells. E2F3, E2F4, E2F5, and E2F6 expressions were positively correlated with the infiltration of $\mathrm{B}$ cells, CD8+ T cells, macrophage, neutrophil, and dendritic cells, and E2F6 expressions were also positively correlated with the infiltration of CD4+ T cells. The expression level of E2F7 was positively correlated with infiltration of CD8 $+\mathrm{T}$ cells and dendritic cells, while it was negatively correlated with infiltration of CD4+ T cells. The expression level of E2F8 was negatively correlated with infiltration of CD4+ T cells, while it was positively correlated with infiltration of $\mathrm{B}$ cells and dendritic cells. The correlation between the expression of E2Fs and the marker genes of PAAD immune cells indicates that E2Fs may be involved in the regulation of PAAD tumor immunity.

In conclusion, our research indicates that the transcription levels of E2F1/3/5/8 are obviously up-regulated in PAAD and may play a significant role in the occurrence and development of PAAD. The high expression of E2F2/3/6/8 can also be used as molecular markers to classify tumor stages in PAAD patients. In addition, abnormal expressions of E2F1/2/3/4/5/7/8 were found to be related with clinical outcomes in patients with PAAD. The study found that E2F1/2/3/5 can be used as potential survival prognostic biomarkers and targets for PAAD. However, the significant correlation between the expression of E2Fs and the infiltration of the six immune cell types in PAAD: B cells, CD8+ $\mathrm{T}$ cells, CD4+ $\mathrm{T}$ cells, macrophages, neutrophils, and dendritic cells, suggests that E2Fs may be involved in the regulation of PAAD tumor immunity. It shows that E2Fs not only can be used as a prognostic indicator of patients with PAAD, but also reflect their immune status. These findings may help better study the molecular basis of PAAD, and may help create more appropriate prognostic tools for PAAD and facilitate the development of new immunotherapeutic. However, more experimental studies are needed to confirm our results, thereby promoting the clinical application of E2Fs as a prognostic indicator or immunotherapy target in PAAD.

\section{DATA AVAILABILITY STATEMENT}

The datasets presented in this study can be found in online repositories. The names of the repository/repositories and accession number(s) can be found in the article/supplementary material.

\section{ETHICS STATEMENT}

The studies involving human participants were reviewed and approved by Medical Ethics Committee of the Hubei University of Medicine-affiliated Taihe Hospital. Written informed consent for participation was not required for this study in accordance with the national legislation and the institutional requirements.

\section{AUTHOR CONTRIBUTIONS}

$\mathrm{X}-\mathrm{SL}, \mathrm{YG}$, and CL conceived the project and wrote the manuscript. X-SL, X-QC, L-MZ, and J-WY participated in data analysis. CL, YG, and X-YK participated in discussion and language editing. Z-JP reviewed the manuscript. All authors contributed to the article and approved the submitted version.

\section{FUNDING}

This work was supported by the Hubei province's Outstanding Medical Academic Leader program, the Foundation for Innovative Research Team of Hubei Provincial Department of Education T2020025, the Hubei Provincial Department of Science and Technology Innovation Group Program (grant no. 2019CFA034), Free-exploring Foundation of Hubei University of Medicine (grant no. FDFR201903), Open Project of Hubei Key Laboratory of Embryonic Stem Cell Research (grant no.2020ESOF009) and the Key Discipline Project of Hubei University of Medicine. 


\section{REFERENCES}

1. Siegel RL, Miller KD, Jemal A. Cancer statistics, 2018. CA: A Cancer J Clin (2018) 68(1):7-30. doi: 10.3322/caac.21442

2. Ilic M, Ilic I. Epidemiology of pancreatic cancer. World J Gastroenterol (2016) 22(44):9694-705. doi: 10.3748/wjg.v22.i44.9694

3. Giannuzzo A, Pedersen SF, Novak I. The P2X7 receptor regulates cell survival, migration and invasion of pancreatic ductal adenocarcinoma cells. Mol Cancer (2015) 14:203. doi: 10.1186/s12943-015-0472-4

4. Rahib L, Smith BD, Aizenberg R, Rosenzweig AB, Fleshman JM, Matrisian LM. Projecting cancer incidence and deaths to 2030: the unexpected burden of thyroid, liver, and pancreas cancers in the United States. Cancer Res (2014) 74(11):2913-21. doi: 10.1158/0008-5472.CAN-14-0155

5. Samuel N, Hudson TJ. The molecular and cellular heterogeneity of pancreatic ductal adenocarcinoma. Nat Rev Gastroenterol Hepatol (2011) 9(2):77-87. doi: 10.1038/nrgastro.2011.215

6. Kirch HC, Putzer B, Schwabe G, Gnauck HK, Schulte HH. Regulation of adenovirus 12 E1A transcription: E2F and ATF motifs in the E1A promoter bind nuclear protein complexes including E2F1, DP-1, cyclin A and/or RB and mediate transcriptional (auto)activation. Cell Mol Biol Res (1993) 39 (8):705-16. doi: 10.1159/000163924

7. Ivey-Hoyle M, Conroy R, Huber HE, Goodhart PJ, Oliff A, Heimbrook DC. Cloning and characterization of E2F-2, a novel protein with the biochemical properties of transcription factor E2F. Mol Cell Biol (1993) 13(12):7802-12. doi: $10.1128 / \mathrm{mcb} \cdot 13.12 .7802$

8. Lees JA, Saito M, Vidal M, Valentine M, Look T, Harlow E, et al. The retinoblastoma protein binds to a family of E2F transcription factors. Mol Cell Biol (1993) 13(12):7813-25. doi: 10.1128/MCB.13.12.7813

9. Ginsberg D, Vairo G, Chittenden T, Xiao ZX, Xu G, Wydner KL, et al. E2F-4, a new member of the E2F transcription factor family, interacts with p107. Gene Dev (1994) 8(22):2665-26. doi: 10.1101/gad.8.22.2665

10. Darville MII, Antoine IV, Mertens-Strijthagen JR, Dupriez VJ, Rousseau GG. An E2F-dependent late-serum-response promoter in a gene that controls glycolysis. Oncogene (1995) 11(8):1509. doi: 10.1002/ajmg.1320590124

11. Maiti B, Li J, de Bruin A, Gordon F, Timmers C, Opavsky R, et al. Cloning and characterization of mouse E2F8, a novel mammalian E2F family membercapable of blocking cellular proliferation. J Biol Chem (2005) 280 (18):18211-20. doi: 10.1074/jbc.M501410200

12. Hagemeier C, Kouzarides T, Bannister AJ, Morkel M, Wenkel J. An E2F-like repressor of transcription. Nature (1997) 390(6660):567-8. doi: 10.1038/ 37507

13. de Bruin A, Maiti B, Jakoi L, Timmers C, Buerki R, Leone G. Identification and characterization of E2F7, a novel mammalian E2F family member capable of blocking cellular proliferation. J Biol Chem (2003) 278(43):42041-9. doi: 10.1074/jbc.M308105200

14. Attwooll C, Denchi EL, Helin K. The E2F family: specific functions and overlapping interests. EMBO J (2004) 23(24):4709-16. doi: 10.1038/ sj.emboj.7600481

15. Ren B. E2F integrates cell cycle progression with DNA repair, replication, and G2/M checkpoints. Gene Dev (2002) 16(2):245-56. doi: 10.1101/gad. 949802

16. Dimova DK, Dyson NJ. The E2F transcriptional network: old acquaintances with new faces. Oncogene (2005) 24(17):2810-26. doi: 10.1038/sj.onc.1208612

17. Zhan L, Huang C, Meng XM, Song Y, Wu XQ, Miu CG, et al. Promising roles of mammalian E2Fs in hepatocellular carcinoma. Cell Signal (2014) 26 (5):1075-81. doi: 10.1016/j.cellsig.2014.01.008

18. Sun C, Li S, Hu W, Zhang J, Zhou Q, Liu C, et al. Comprehensive Analysis of the Expression and Prognosis for E2Fs in Human BreastCancer. Mol Ther J Am Soc Gene Ther (2019) 27(6):1153-65. doi: 10.1016/j.ymthe.2019.03.019

19. Zhou Q, Zhang F, He Z, Zuo M. E2F2/5/8 Serve as Potential Prognostic Biomarkers and Targets for Human OvarianCancer. Front Oncol (2019) 9:161. doi: 10.3389/fonc.2019.00161

20. $\mathrm{Yu} \mathrm{H}, \mathrm{Li} \mathrm{Z}$, Wang M. Expression and prognostic role of E2F transcription factors in high-grade glioma. CNS Neurosci Ther (2020) 26:741-53. doi: $10.1111 / \mathrm{cns} .13295$

21. Huang YL, Ning G, Chen LB, Lian YF, Gu YR, Wang JL, et al. Promising diagnostic and prognostic value of E2Fs in human hepatocellular carcinoma. Cancer Manag Res (2019) 11:1725-40. doi: 10.2147/CMAR.S182001
22. Sun F, Lin Y, Li S, Gao J, Han B, Zhang C. MiR-210 knockdown promotes the development of pancreatic cancer via upregulating E2F3 expression. Eur Rev Med Pharmaco (2018) 22(24):8640-8. doi: 10.26355/eurrev_201812_16628

23. Lin C, Hu Z, Yuan G, Su H, Zeng Y, Guo Z, et al. MicroRNA-1179 inhibits the proliferation, migration and invasion of humanpancreatic cancer cells by targeting E2F5. Chem Biol Interact (2018) 291:65-71. doi: 10.1016/ j.cbi.2018.05.017

24. Sealfon SC, Chu TT. RNA and DNA microarrays. Methods Mol Biol (Clifton NJ) (2011) 671(671):3. doi: 10.1007/978-1-59745-551-0_1

25. Raghavachari N, Barb J, Yang Y, Liu P, Woodhouse K, Levy D, et al. A systematic comparison and evaluation of high density exon arrays and RNAseq technology used to unravel the peripheral blood transcriptome of sickle cell disease. BMC Med Genomics (2012) 5(1):28. doi: 10.1186/1755-8794-5-28

26. Rhodes DR, Yu J, Shanker K, Deshpande N, Varambally R, Ghosh D, et al. ONCOMINE: A Cancer Microarray Database and Integrated Data-Mining Platform. Neoplasia (2004) 6(1):1-6. doi: 10.1016/S1476-5586(04)80047-2

27. Rhodes DR, Kalyana-Sundaram S, Mahavisno V, Varambally R, Yu J, Briggs $\mathrm{BB}$, et al. Oncomine 3.0: genes, pathways, and networks in a collection of 18,000 cancer geneexpression profiles. Neoplasia (New York NY) (2007) 9 (2):166-80. doi: 10.1593/neo.07112

28. Tang Z, Li C, Kang B, Gao G, Li C, Zhang Z. GEPIA: a web server for cancer and normal gene expression profiling and interactive analyses. Nucleic Acids Res (2017) 45(W1):W98-W102. doi: 10.1093/nar/gkx247

29. Gyorffy B, Surowiak P, Budczies J, Lanczky A. Online survival analysis software to assess the prognostic value of biomarkers using transcriptomic data in non-small-cell lung cancer. PLoS One (2013) 8(12):e82241. doi: 10.1371/journal.pone.0082241

30. Nagy A, Lanczky A, Menyhart O, Gyorffy B. Validation of miRNA prognostic power in hepatocellular carcinoma using expressiondata of independent datasets. Sci Rep UK (2018) 8(1):9227. doi: 10.1038/s41598-018-27521-y

31. Tomczak K, Czerwińska P, Wiznerowicz M. Review The Cancer Genome Atlas (TCGA): an immeasurable source of knowledge. Współczesna Onkol (2015) 1A(1A):68-77. doi: 10.5114/wo.2014.47136

32. Gao J, Aksoy BA, Dogrusoz U, Dresdner G, Gross B, Sumer SO, et al. Integrative analysis of complex cancer genomics and clinical profiles using the cBioPortal. Sci Signal (2013) 6(269):pl1-1. doi: 10.1126/scisignal.2004088

33. Cerami E, Gao J, Dogrusoz U, Gross BE, Sumer SO, Aksoy BA, et al. The cBio cancer genomics portal: an open platform for exploring multidimensional cancer genomics data. Cancer Discov (2012) 2(5):401-4. doi: 10.1158/21598290.CD-12-0095

34. Warde-Farley D, Donaldson SL, Comes O, Zuberi K, Badrawi R, Chao P, et al. The GeneMANIA prediction server: biological network integration for geneprioritization and predicting gene function. Nucleic Acids Res (2010) 38 (Web Server issue):W214-20. doi: 10.1093/nar/gkq537

35. Szklarczyk D, Gable AL, Lyon D, Junge A, Wyder S, Huerta-Cepas J, et al. STRING v11: protein-protein association networks with increased coverage, supporting functional discovery in genome-wide experimental datasets. Nucleic Acids Res (2019) 47(D1):D607-13. doi: 10.1093/nar/gky1131

36. Zhou Y, Zhou B, Pache L, Chang M, Khodabakhshi AH, Tanaseichuk O, et al. Metascape provides a biologist-oriented resource for the analysis ofsystems-level datasets. Nat Commun (2019) 10(1):1523. doi: 10.1038/s41467-019-09234-6

37. Li T, Fan J, Wang B, Traugh N, Chen Q, Liu JS, et al. TIMER: A Web Server for Comprehensive Analysis of Tumor-Infiltrating ImmuneCells. Cancer Res (2017) 77(21):e108-10. doi: 10.1158/0008-5472.CAN-17-0307

38. Buchholz M, Braun M, Heidenblut A, Kestler HA, Kloppel G, Schmiegel W, et al. Transcriptome analysis of microdissected pancreatic intraepithelial neoplasticlesions. Oncogene (2005) 24(44):6626-36. doi: 10.1038/ sj.onc. 1208804

39. Logsdon CD, Simeone DM, Binkley C, Arumugam T, Greenson JK, Giordano TJ, et al. Molecular profiling of pancreatic adenocarcinoma and chronic pancreatitis identifies multiple genes differentially regulated in pancreatic cancer. Cancer Res (2003) 63(10):2649-57. doi: 10.1053/gast.2002.00000

40. Segara D, Biankin AV, Kench JG, Langusch CC, Dawson AC, Skalicky DA, et al. Expression of HOXB2, a retinoic acid signaling target in pancreatic cancer andpancreatic intraepithelial neoplasia. Clin Cancer Res (2005) 11 (9):3587-96. doi: 10.1158/1078-0432.CCR-04-1813

41. Badea L, Herlea V, Dima SO, Dumitrascu T, Popescu I. Combined gene expression analysis of whole-tissue and microdissected pancreatic ductal 
adenocarcinoma identifies genes specifically overexpressed in tumor epithelia. Hepatogastroenterology (2008) 55(88):2016-27.

42. Grutzmann R, Pilarsky C, Ammerpohl O, Luttges J, Bohme A, Sipos B, et al. Gene expression profiling of microdissected pancreatic ductal carcinomas using high-density DNA microarrays. Neoplasia (2004) 6(5):611-22. doi: 10.1593/neo.04295

43. Ishikawa M, Yoshida K, Yamashita Y, Ota J, Takada S, Kisanuki H, et al. Experimental trial for diagnosis of pancreatic ductal carcinoma based on geneexpression profiles of pancreatic ductal cells. Cancer Sci (2005) 96 (7):387-93. doi: 10.1111/j.1349-7006.2005.00064.x

44. Pei H, Li L, Fridley BL, Jenkins GD, Kalari KR, Lingle W, et al. FKBP51 affects cancer cell response to chemotherapy by negatively regulating Akt. Cancer Cell (2009) 16(3):259-66. doi: 10.1016/j.ccr.2009.07.016

45. Emmrich S, Pützer BM. Checks and balances: E2F - MicroRNA crosstalk in cancer control. Cell Cycle (2010) 9(13):2555-67. doi: 10.4161/ cc.9.13.12061

46. Zhan L, Zhang Y, Wang W, Song E, Fan Y, Wei B. E2F1: a promising regulator in ovarian carcinoma. Tumor Biol (2016) 37(3):2823-31. doi: 10.1007/s13277015-4770-7

47. Ma L, Tian X, Wang F, Zhang Z, Du C, Xie X, et al. The long noncoding RNA H19 promotes cell proliferation via E2F-1 in pancreaticductal adenocarcinoma. Cancer Biol Ther (2016) 17(10):1051-61. doi: 10.1080/ 15384047.2016.1219814
48. Jiang H, Martin V, Alonso M, Gomez-Manzano C, Fueyo-Margareto J. RBE2F1: Molecular rheostat for autophagy and apoptosis. Autophagy (2010) 6 (8):1216-7. doi: 10.4161/auto.6.8.13695

49. Infante A, Laresgoiti U, Fernández-Rueda J, Fullaondo A, Galán J, DíazUriarte R, et al. E2F2 represses cell cycle regulators to maintain quiescence. Cell Cycle (2008) 7(24):3915-27. doi: 10.4161/cc.7.24.7379

50. Yao Z, Chen Q, Ni Z, Zhou L, Wang Y, Yang Y, et al. Long Non-Coding RNA Differentiation Antagonizing Nonprotein Coding RNA (DANCR) Promotes Proliferation and Invasion of Pancreatic Cancer by Sponging miR-214-5p to Regulate E2F2 Expression. Med Sci Monitor (2019) 25:4544-52. doi: 10.12659/ MSM. 916960

Conflict of Interest: The authors declare that the research was conducted in the absence of any commercial or financial relationships that could be construed as a potential conflict of interest.

Copyright $\odot 2021$ Liu, Gao, Liu, Chen, Zhou, Yang, Kui and Pei. This is an openaccess article distributed under the terms of the Creative Commons Attribution License (CC BY). The use, distribution or reproduction in other forums is permitted, provided the original author(s) and the copyright owner(s) are credited and that the original publication in this journal is cited, in accordance with accepted academic practice. No use, distribution or reproduction is permitted which does not comply with these terms. 